\title{
Kohn-Luttinger superconductivity in graphene
}

\author{
J. González \\ Instituto de Estructura de la Materia, Consejo Superior de Investigaciones Científicas, Serrano 123, 28006 Madrid, Spain
}

(Received 11 August 2008; revised manuscript received 18 October 2008; published 24 November 2008)

\begin{abstract}
We investigate the development of superconductivity in graphene when the Fermi level becomes close to one of the Van Hove singularities of the electron system. The origin of the pairing instability lies in the strong anisotropy of the $e-e$ scattering at the Van Hove filling, which leads to a channel with attractive coupling when making the projection of the BCS vertex on the symmetry modes with nontrivial angular dependence along the Fermi line. We show that the scale of the superconducting instability may be pushed up to temperatures larger than $10 \mathrm{~K}$, depending on the ability to tune the system to the proximity of the Van Hove singularity.
\end{abstract}

DOI: 10.1103/PhysRevB.78.205431

PACS number(s): 81.05.Uw, 74.20.Mn, 74.78.-w

\section{INTRODUCTION}

Since the fabrication in 2004 of single atomic layers of carbon, this material (so-called graphene) has been attracting a lot of attention. ${ }^{1}$ The undoped system has conical valence and conduction bands meeting at two different Fermi points (known as Dirac points). ${ }^{2,3}$ This peculiar dispersion has shown to be at the origin of a number of remarkable effects, such as the existence of a minimum conductivity at the charge neutrality point. ${ }^{4-7}$

From the point of view of possible applications, the interest in graphene has been driven by the large electron mobilities attained in typical experimental samples. Another remarkable property is that graphene can be used to build Josephson junctions when placed between superconducting contacts. ${ }^{8}$ It then becomes quite intriguing whether graphene may support superconducting correlations on its own under suitable experimental conditions. On theoretical grounds, it is known that a model based on the conical dispersion requires a minimum strength of the pairing interaction for the development of a superconducting instability in the undoped system. ${ }^{9}$ There have been already several proposals to drive graphene toward a pairing instability upon doping, placing the emphasis on the role of topological defects, ${ }^{10}$ the effect of a metallic coating, ${ }^{11}$ or the possibility of inducing superconductivity by electronic correlations. ${ }^{12,13}$

In this paper we investigate a different route to superconductivity in graphene when the Fermi level is close to one of the Van Hove singularities (VHSs) of the electron system. These are points characterized by a divergent density of states, which has the effect of enhancing the magnetic and superconducting correlations. ${ }^{14}$ The origin of the pairing instability lies in the strong anisotropy of the Fermi line at the Van Hove filling, and it proceeds following in essence the same mechanism proposed by Kohn and Luttinger ${ }^{15}$ to show that superconductivity can arise out of purely repulsive interactions. This is possible as long as the $e-e$ scattering becomes highly anisotropic so that a channel with attractive coupling may appear when making the projection on the symmetry modes with nontrivial angular dependence over the Fermi surface.

\section{TIGHT-BINDING MODEL}

In the case of graphene, there are two VHSs located at about $3 \mathrm{eV}$ above and below the Dirac points in the spec- trum. Each of the singularities correspond to the existence of three inequivalent saddle points of the dispersion at the boundary of the Brillouin zone, as shown in Fig. 1. In order to find the dominant electronic instability arising from the divergent density of states, one has to determine first the shape of the Fermi line when the Fermi level is close to the VHS. For this purpose, we have characterized the energy contour lines around the saddle points of the valence band by means of a tight-binding model, suited to fit the dispersion $\varepsilon(\mathbf{k})$ known from angle-resolved photoemission spectroscopy (ARPES). ${ }^{16}$ In the model, we have considered the transfer integrals for first, second, and third neighbors of the graphene lattice, labeled by $t, d$, and $t^{\prime}$, respectively, and the overlap integral $s$ between first neighbors. In terms of these parameters and setting the lattice spacing $a=1$, the Fermi velocity at the Dirac points is given by $v_{F}=(3 / 2)\left(t-2 t^{\prime}\right.$ $+3 s d$ ), which according to the value found in graphene implies that $t-2 t^{\prime}+3 s d \approx 2.7 \mathrm{eV}$. Moreover, the level of the

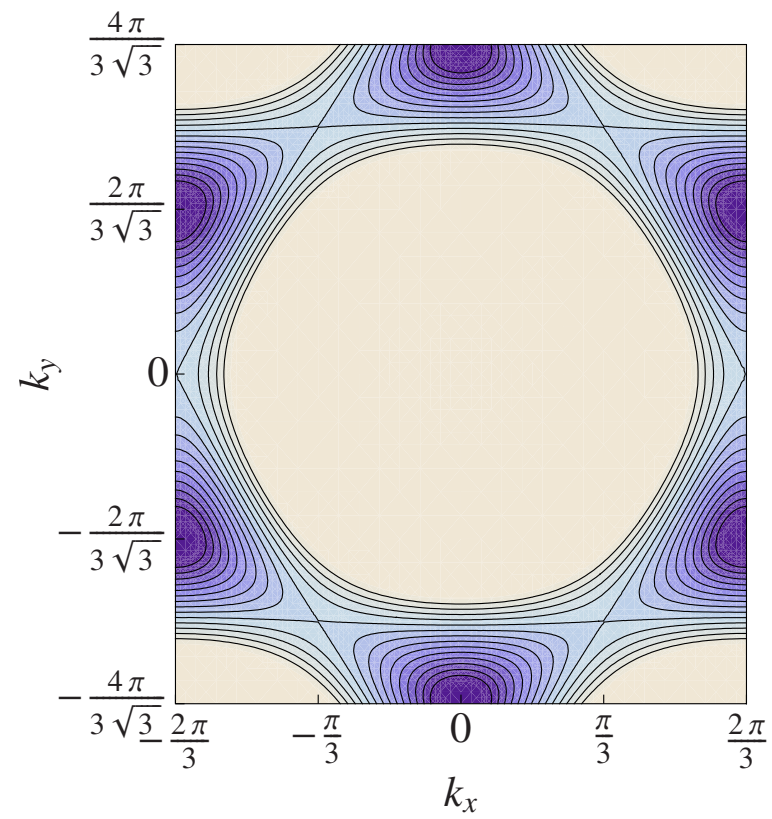

FIG. 1. (Color online) Plot of energy contour lines around the saddle points of the valence band of graphene, obtained from a tight-binding model with first-, second-, and third-neighbor hopping parameters given by Eqs. (1)-(3) for $s=0.1$. 


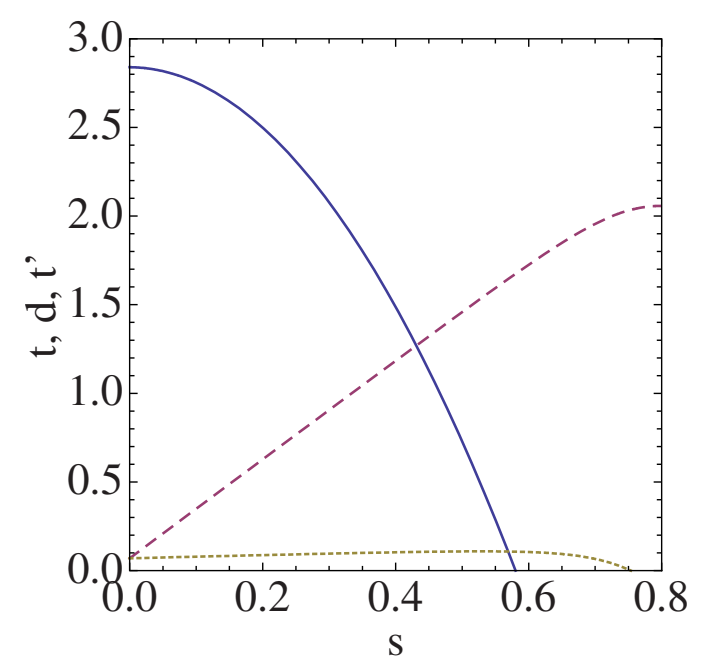

FIG. 2. (Color online) Plot of the hopping parameters $t$ (full line), $d$ (dashed line), and $t^{\prime}$ (dotted line) given in $\mathrm{eV}$ units as functions of the overlap integral $s$ and satisfying the constraints [Eqs. (1)-(3)].

saddle points relative to the Dirac points turns out to be $3 d$ $+\left(t-3 t^{\prime}-2 d\right) /(1+s)$, which must correspond to the value of $\approx 2.7 \mathrm{eV}$ found in ARPES. ${ }^{16}$ With this input, we arrive at two conditions (with $t, d$, and $t^{\prime}$ measured in $\mathrm{eV}$ ):

$$
\begin{gathered}
t^{\prime} \approx d-2.7 s, \\
t \approx 2.7+2 d-5.4 s-3 s d .
\end{gathered}
$$

Finally, we can adjust the parameters to reproduce the curvature of the dispersion at the saddle points, ${ }^{16}$ arriving at a dependence of the second-neighbor hopping $d$ which is linear on $s$ to very high accuracy,

$$
d \approx 0.07+2.8 s+O\left(s^{2}\right) .
$$

The ARPES dispersion around the saddle points can then be fitted leaving the overlap integral $s$ free. This makes the different transfer integrals dependent on that parameter, as represented in Fig. 2. The important point is that the thirdneighbor hopping $t^{\prime}$ remains always constrained to a very small value, $t^{\prime} \approx 0.1 \mathrm{eV}$. This parameter controls the approximate nesting of the Fermi line, that is, the possibility of having regions in which the dispersion satisfies $\varepsilon(\mathbf{k}) \approx$ $-\varepsilon(\mathbf{Q}+\mathbf{k})$, with a fixed momentum $\mathbf{Q}$. This is realized in the model with hopping parameters obtained from Eqs. (1)-(3) when $\mathbf{Q}$ is the momentum connecting two inequivalent saddle points, as observed in a typical plot of energy contour lines shown in Fig. 1. The exact condition of nesting is met in the model with $t^{\prime}=0$ while the relative small values that we find for this parameter still lead to quite straight Fermi lines connecting the saddle points, especially in the case of the valence band.

The measure of the nesting is given by the susceptibility $\chi(\mathbf{Q}, \omega)$, which diverges in any event in the proximity to the singular density of states. We may approximate for instance the dispersion with the deviation $\delta \mathbf{k}$ from the saddle point of the valence band at $(2 \pi / 3,0)$ by

$$
\varepsilon(\mathbf{k}) \approx-\alpha \delta k_{x}^{2}+\beta \delta k_{y}^{2}
$$

Assuming that the VHS is in general at an energy $\mu$ from the Fermi level, we have

$$
\chi(\mathbf{Q}, \omega) \approx \frac{1}{2 \sqrt{3} \pi^{2}} \frac{c^{\prime}}{\alpha+\beta} \log \left(\frac{\Lambda_{0}}{\omega+\mu}\right),
$$

where $\Lambda_{0}$ is an upper cutoff in the description of the saddle points, which we take as $\approx 1 \mathrm{eV}$. The nesting instability at $\alpha=3 \beta$ appears in the dependence

$$
c^{\prime}=\log \left(\frac{1+\sqrt{\beta / 3 \alpha}}{1-\sqrt{\beta / 3 \alpha}}\right)+\log \left(\frac{1+\sqrt{3 \beta / \alpha}}{1-\sqrt{3 \beta / \alpha}}\right) .
$$

This has to be confronted with the susceptibility at vanishing momentum transfer, which is

$$
\chi(\mathbf{0}, \omega) \approx \frac{1}{4 \pi^{2}} \frac{1}{\sqrt{\alpha \beta}} \log \left(\frac{\Lambda_{0}}{\omega+\mu}\right) .
$$

For the VHS in the valence band of graphene, sensible values of the parameters are $\alpha \approx 7.41$ and $\beta \approx 2.03$ (as obtained for $s=0.1$ ), which give $c^{\prime} \approx 3.6$. We see therefore that the susceptibility at momentum $\mathbf{Q}$ is about two times larger than that at vanishing momentum.

The main physical effect of the nesting of the Fermi line is to enhance the anisotropy of the $e-e$ scattering. In this respect, the accurate determination of the shape of the Fermi line is an important factor in discerning whether such an anisotropy may be strong enough to induce a pairing instability. We will see that this happens when the response of the electron system at momentum $\mathbf{Q}$ prevails over that at vanishing momentum, that is, when $2 \chi(\mathbf{Q}, \omega)>3 \chi(\mathbf{0}, \omega) .{ }^{17}$ This condition is satisfied for the VHS in the valence band, according to our parametrization of the tight-binding model. The values of $\alpha$ and $\beta$ are actually quite insensitive to variations in the overlap integral $s$ in the range of $0.1-0.3$, taking into account the corresponding changes in the hopping parameters given by Eqs. (1)-(3). The effects derived from the anisotropy of the $e$ - $e$ scattering appear to be then quite robust in the case of the VHS in the valence band of graphene.

A similar analysis carried out for the VHS in the conduction band leads however to a less clear situation. The dispersion can be approximated then around the saddle point at $(2 \pi / 3,0)$ by taking parameters $\widetilde{\alpha}$ and $\widetilde{\beta}$ such that

$$
\varepsilon(\mathbf{k}) \approx \widetilde{\alpha} \delta k_{x}^{2}-\tilde{\beta} \delta k_{y}^{2} .
$$

In principle, a quite precise determination of $\widetilde{\alpha}$ and $\widetilde{\beta}$ is not possible now as the above evaluation of the hopping parameters was made to achieve an accurate fit to the valence band observed in the ARPES experiments. Anyhow, the use of the same constraints [Eqs. (1)-(3)] does not give rise in this case to the required anisotropy in the $e-e$ interaction. By varying the overlap integral $s$ in the range of $0.1-0.3$, we observe corresponding changes in $\widetilde{\alpha}$ from 1.75 to 1.53 while those in $\widetilde{\beta}$ are from 6.86 to 7.12 . By taking pairs of respective values of these two parameters in that range, we observe that the combination $2 \chi(\mathbf{Q}, \omega)-3 \chi(\mathbf{0}, \omega)$ is now either negative, or positive but too small, to give rise to a significant effect. 
Therefore, we will not pursue in what follows the study of the VHS in the conduction band of graphene while we concentrate on the analysis of the effects derived from the VHS in the valence band, allowing us to reach definite predictions.

\section{PAIRING INSTABILITY}

When the Fermi line becomes close to the saddle points, the anisotropy of the $e-e$ scattering may actually induce a pairing instability. This can be understood by re-elaborating in the present context the argument given by Kohn and Luttinger $^{15}$ a long time ago. We denote the interaction vertex by $V\left(\theta, \theta^{\prime}\right)$ for the particular case of BCS kinematics, in which the incoming (outgoing) particles collide with zero total spin and zero total momentum, and the angle $\theta\left(\theta^{\prime}\right)$ locates the position of the spin-up particle over the Fermi line. The BCS vertex gets corrections at low energies by the effect of the high-energy electron modes in slices between energies $\Lambda$ and $\Lambda+d \Lambda$ about the Fermi level. ${ }^{18}$ The integration of these modes gives the variation

$$
d V\left(\theta, \theta^{\prime}\right)=\frac{d \Lambda}{\Lambda} \int_{0}^{2 \pi} \frac{d \theta^{\prime \prime}}{(2 \pi)^{2}} \frac{\partial k_{\|}}{\partial \theta^{\prime \prime}} \frac{1}{v\left(\theta^{\prime \prime}\right)} V\left(\theta, \theta^{\prime \prime}\right) V\left(\theta^{\prime \prime}, \theta^{\prime}\right),
$$

where $v\left(\theta^{\prime}\right)$ is the gradient of the dispersion and $\partial k_{\|} / \partial \theta^{\prime \prime}$ is the variation in the momentum along the slice parametrized by the angle $\theta^{\prime \prime}$. We can write the above equation in more compact form by passing to the variable

$$
\phi(\theta)=\frac{1}{2 \pi n(\Lambda)} \int_{0}^{\theta} d \theta^{\prime \prime} \frac{\partial k_{\|}}{\partial \theta^{\prime \prime}} \frac{1}{v\left(\theta^{\prime \prime}\right)},
$$

where the density of states $n(\Lambda)$ is introduced so that the new variable also ranges from 0 to $2 \pi .{ }^{19}$ After defining the transformed vertex by $\tilde{V}\left(\phi, \phi^{\prime}\right)=V\left(\theta, \theta^{\prime}\right)$, we get

$$
\frac{\partial \tilde{V}\left(\phi, \phi^{\prime}\right)}{\partial \log \Lambda}=\frac{n(\Lambda)}{2 \pi} \int_{0}^{2 \pi} d \phi^{\prime \prime} \tilde{V}\left(\phi, \phi^{\prime \prime}\right) \tilde{V}\left(\phi^{\prime \prime}, \phi^{\prime}\right) .
$$

We can further decompose the vertex $\tilde{V}\left(\phi, \phi^{\prime}\right)$ in terms of the eigenmodes $\Psi_{m}^{\gamma}(\phi)$ for the different representations $\gamma$ of the point symmetry group,

$$
\tilde{V}\left(\phi, \phi^{\prime}\right)=\sum_{\gamma, m, n} V_{m, n}^{\gamma} \Psi_{m}^{\gamma}(\phi) \Psi_{n}^{\gamma}\left(\phi^{\prime}\right) .
$$

We obtain then the set of coupled scaling equations

$$
\frac{\partial V_{m, n}^{\gamma}}{\partial \log \Lambda}=n(\Lambda) \sum_{s} V_{m, s}^{\gamma} V_{s, n}^{\gamma}
$$

In this framework, we recover the analog of the KohnLuttinger mechanism when any of the couplings $V_{m, n}^{\gamma}$ turns out to be negative in such a way that an unstable flow develops as the Fermi level is approached in the low-energy limit $\Lambda \rightarrow 0$.

We remark that the scaling equation (11) encodes the corrections to the BCS vertex that are logarithmically divergent at low energies in the particle-particle channel. If we start

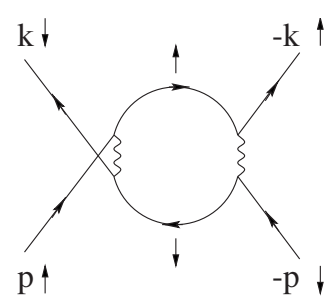

(a)

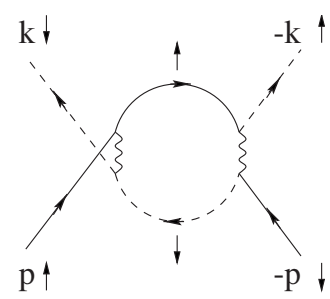

(b)
FIG. 3. Lowest-order particle-hole corrections to the BCS vertex for a bare on-site interaction. Full and dashed lines represent the propagation of electrons around different saddle points.

solving the scaling equation at an intermediate energy scale $\Lambda$, the initial values of the function $\tilde{V}\left(\phi, \phi^{\prime}\right)$ will be dictated by the bare interaction as well as by finite corrections to it, given in general by diagrams in the particle-hole channel. Previous scaling analyses of electrons near a VHS have shown that only the momentum-independent component of the interaction potential is not irrelevant at low energies, ${ }^{20}$ which is consistent with the large screening effects from the divergent density of states. For this reason, we may consider that a bare on-site repulsion $U$ between the electron densities of opposite spin provides a sensible form of interaction close to the Van Hove filling. The relevant point is that the bare short-range interaction gives rise to particle-hole corrections to the BCS vertex like those represented in Fig. 3. If we measure the angles $\phi$ and $\phi^{\prime}$ with respect to the $x$ axis, we observe for instance that the value of $\tilde{V}(0, \pi / 3)$ will be enhanced by the particle-hole susceptibility (5) at momentum Q while $\tilde{V}(0,0)$ is enhanced by the particle-hole susceptibility (7) at zero momentum. The prevalence of $\chi(\mathbf{Q}, \omega)$ implies that

$$
\tilde{V}(0,0)-\tilde{V}(0, \pi / 3) \sim 3 U^{2} \chi(\mathbf{0}, \omega)-2 U^{2} \chi(\mathbf{Q}, \omega)<0 .
$$

In general, we can anticipate the dominant terms in the modulation of the BCS vertex complying with the symmetry of the Fermi line:

$$
\begin{gathered}
\tilde{V}(\phi, \phi) \approx c_{0}+c_{6} \cos (6 \phi)+\ldots, \\
\tilde{V}(0, \phi) \approx c_{0}^{\prime}+c_{2}^{\prime} \cos (2 \phi)+c_{4}^{\prime} \cos (4 \phi)+c_{6}^{\prime} \cos (6 \phi)+\ldots,
\end{gathered}
$$

$$
\begin{aligned}
\tilde{V}(\phi, \pi-\phi) \approx & c_{0}^{\prime \prime}+c_{2}^{\prime \prime} \cos (2 \phi)+c_{4}^{\prime \prime} \cos (4 \phi)+c_{6}^{\prime \prime} \cos (6 \phi) \\
& +\ldots
\end{aligned}
$$

As we will see, several relations can be obtained between the coefficients in Eqs. (15)-(17) by estimating the strength of the different scattering processes around the Fermi line.

The Fourier expansions (15)-(17) match well with the decomposition (12) of the BCS vertex in terms of the basis functions $\Psi_{n}^{\gamma}(\phi)$. The point symmetry group is $C_{6 v}$, which has six irreducible representations. Four of them are one dimensional, with respective sets of basis functions given by 
$\{\cos (6 n \phi)\}, \quad\{\sin (6 n \phi)\}, \quad\{\cos [(6 n+3) \phi]\}, \quad$ and $\quad\{\sin [(6 n$ $+3) \phi]\}$ ( $n$ being always an integer). The other two representations are two dimensional and have sets of basis functions which can be represented by $\{\cos (m \phi), \sin (m \phi)\}$, with the integer $m$ running over all values that are not multiples of 3 and which are odd for one of the representations and even for the other. We can write therefore an expansion of the BCS vertex following Eq. (12) and matching the angular dependence in Eqs. (15)-(17),

$$
\begin{aligned}
\tilde{V}\left(\phi, \phi^{\prime}\right)= & V_{0,0}+\sqrt{2} V_{0,6}\left[\cos (6 \phi)+\cos \left(6 \phi^{\prime}\right)\right] \\
& +2 V_{2,2}\left[\cos (2 \phi) \cos \left(2 \phi^{\prime}\right)+\sin (2 \phi) \sin \left(2 \phi^{\prime}\right)\right] \\
& +2 V_{2,4}\left[\cos (2 \phi) \cos \left(4 \phi^{\prime}\right)-\sin (2 \phi) \sin \left(4 \phi^{\prime}\right)\right. \\
& \left.+\phi \leftrightarrow \phi^{\prime}\right]+2 V_{3,3} \cos (3 \phi) \cos \left(3 \phi^{\prime}\right) \\
& +2 V_{3,3}^{\prime} \sin (3 \phi) \sin \left(3 \phi^{\prime}\right)+\ldots
\end{aligned}
$$

One can check that the terms displayed in Eq. (18) account for the modulation of the BCS vertex in Eqs. (15)-(17). We identify actually the different coefficients $c_{2}^{\prime}$ $=2 V_{2,2}+2 V_{2,4}, c_{4}^{\prime}=2 V_{2,4}$, and $c_{6}^{\prime}=\sqrt{2} V_{0,6}$, on one hand, and $c_{2}^{\prime \prime}=4 V_{2,4}, \quad c_{4}^{\prime \prime}=2 V_{2,2}$, and $c_{6}^{\prime \prime}=2 \sqrt{2} V_{0,6}-V_{3,3}^{\prime}$, on the other hand. The constraint (14) can be translated then to these couplings since $\widetilde{V}(0,0)-\widetilde{V}(0, \pi / 3)$ is given by the combination $3\left(c_{2}^{\prime}+c_{4}^{\prime}\right) / 2=3\left(c_{2}^{\prime \prime}+c_{4}^{\prime \prime}\right) / 2$. We find

$$
3\left(V_{2,2}+2 V_{2,4}\right) \sim 3 U^{2} \chi(\mathbf{0}, \omega)-2 U^{2} \chi(\mathbf{Q}, \omega) .
$$

This already points at the existence of an unstable flow in the channel corresponding to the representation with $d$-wave symmetry, as long as $3 \chi(\mathbf{0}, \omega)<2 \chi(\mathbf{Q}, \omega)$. A closer inspection reveals actually that both couplings $V_{2,2}$ and $V_{2,4}$ must be negative. To show this, one more constraint can be enforced by noticing that $\widetilde{V}(\pi / 6,5 \pi / 6)-\widetilde{V}(\pi / 2, \pi / 2)=3\left(c_{2}^{\prime \prime}\right.$ $\left.-c_{4}^{\prime \prime}\right) / 2$. The relevant point is that the particle-hole corrections to the BCS vertex at those angles are not singular near the VHS as they involve scattering processes that scale at most as $\sim \sqrt{\omega} .{ }^{21}$ Therefore, the dominant contribution to both couplings $V_{2,2}$ and $V_{2,4}$ arises from the logarithmic dependence on $\omega$ at the right-hand side of Eq. (19).

As long as $V_{2,2}$ and $V_{2,4}$ are negative, we have a pairing instability in the system whose critical scale can be estimated by solving the coupled scaling equations (13) in the relevant symmetry channel. We can truncate the set of equations to

$$
\frac{\partial}{\partial \log \Lambda}\left(\begin{array}{ll}
V_{2,2} & V_{2,4} \\
V_{4,2} & V_{4,4}
\end{array}\right) \approx n(\Lambda)\left(\begin{array}{ll}
V_{2,2} & V_{2,4} \\
V_{4,2} & V_{4,4}
\end{array}\right)\left(\begin{array}{ll}
V_{2,2} & V_{2,4} \\
V_{4,2} & V_{4,4}
\end{array}\right) .
$$

Equation (20) can be easily solved by passing to the eigenvalues $\lambda_{1}$ and $\lambda_{2}$ of the matrix of couplings. We recall that $V_{4,4}$ does not appear at the dominant level in the expansion (18) of the BCS vertex. The two eigenvalues can be approximated then by $\lambda_{1,2}=\left(V_{2,2} \pm \sqrt{V_{2,2}^{2}+4 V_{2,4}^{2}}\right) / 2$. It is clear that the positive eigenvalue $\lambda_{1}$ vanishes in the low-energy limit while the signature of the pairing instability is given by the growth of $\lambda_{2}$ toward very large negative values as $\Lambda \rightarrow 0$.

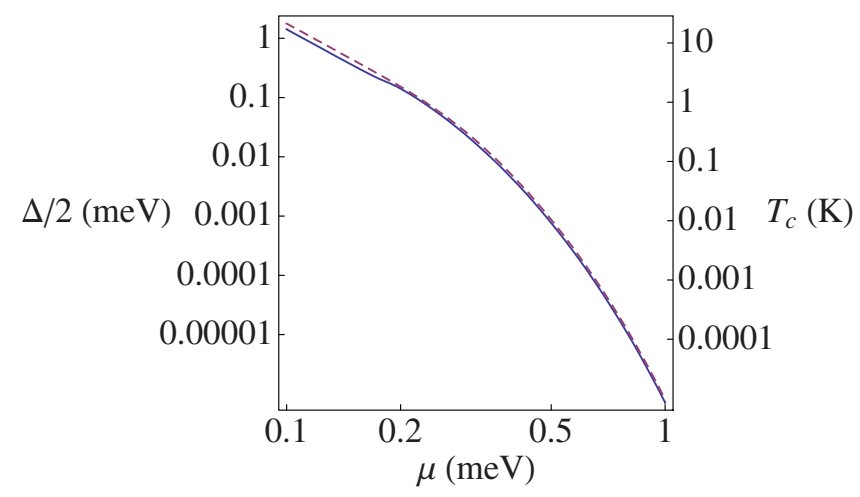

FIG. 4. (Color online) Plot of the scale $\Delta / 2$ at which the eigenvalue $\lambda_{2}$ diverges (full line) and the critical temperature $T_{c}$ (dashed line), as a function of the deviation $\mu$ of the VHS from the Fermi level.

\section{SUPERCONDUCTING GAP AND CRITICAL TEMPERATURE}

We determine the onset of the pairing instability in terms of the energy $\Lambda_{c}$ at which the solution of Eq. (20) diverges. In our scaling approach, the progressive integration of electron modes stops at that point, which can be identified with the opening of a gap in the single-particle spectrum. According to this argument, the magnitude $\Delta$ of the gap can be computed as $\Delta=2 \Lambda_{c}$, which turns out to be also consistent with the determination of the critical temperature carried out in what follows.

To find the behavior of the negative eigenvalue $\lambda_{2}$, we take for $n(\Lambda)$ the density of states about the VHS

$$
n(\varepsilon) \approx \frac{3}{4 \pi^{2}} \frac{1}{\sqrt{\alpha \beta}} \log \left(\frac{\Lambda_{0}}{|\varepsilon+\mu|}\right) .
$$

The other important factor in the resolution of Eq. (20) is the choice of initial conditions for $V_{2,2}$ and $V_{2,4}$ at the upper cutoff $\Lambda_{0}$. In this respect, we have taken a value of $U$ $=4 \mathrm{eV}$ for the bare on-site repulsion, which is between the estimates made for graphite and carbon nanotubes. ${ }^{22}$ In order to go beyond the perturbative particle-hole corrections to the BCS vertex, we have summed up the series of leading logarithms obtained by iteration of the particle-hole susceptibilities in the diagrams of Fig. 3. Thus, we have constrained the initial couplings by the condition

$$
3\left(V_{2,2}+2 V_{2,4}\right)=\frac{U}{1-3 U \chi(\mathbf{0}, \mu)}-\frac{U}{1-2 U \chi(\mathbf{Q}, \mu)} .
$$

The precise values of $V_{2,2}$ and $V_{2,4}$ have been obtained by adding the other constraint mentioned below Eq. (19), which $\operatorname{reads} 3\left(2 V_{2,4}-V_{2,2}\right)=\tilde{V}(\pi / 6,5 \pi / 6)-\tilde{V}(\pi / 2, \pi / 2)$.

The results obtained for the scale $\Lambda_{c}$ at which $\lambda_{2}$ diverges are shown in Fig. 4. We observe that the scale of the pairing instability depends drastically on the value of the chemical potential $\mu$ measuring the deviation of the VHS from the Fermi level. The plot of Fig. 4 shows anyhow that the instability exists irrespective of the value of $\mu$. We recall in this respect that the Kohn-Luttinger mechanism was proposed to put forward the idea that any Fermi liquid is unstable at 
sufficiently low temperature. ${ }^{15}$ On the other hand, an inflection point can be seen in the plot of Fig. 4 for a value of $\mu$ slightly below $0.2 \mathrm{meV}$. That feature corresponds to the case in which the scale $\Lambda_{c}$ for the opening of the gap coincides with the deviation $\mu$ of the VHS from the Fermi level. Values of $\mu$ to the right of the inflection point correspond to the regime where the VHS lies outside the superconducting gap while the values to the left are points where the gap develops around the VHS.

An important question is the determination of the finite temperature effects on the pairing instability. This analysis is particularly interesting in our electron system as the strength of the instability is dictated by the proximity to the VHS while the thermal effects have the ability to smear out the superconducting correlations at low energies. We can incorporate such effects by computing the BCS vertex within the many-body formalism at temperature $T \neq 0$. The Green's function for electrons with saddle-point dispersion $\varepsilon(\mathbf{k})$ reads then as ${ }^{23}$

$$
G^{(0)}(\mathbf{k}, \omega)=P \frac{1}{\omega-\varepsilon(\mathbf{k})}-i \pi \tanh \left(\frac{\omega}{2 k_{B} T}\right) \delta(\omega-\varepsilon(\mathbf{k})) .
$$

Taking into account the change in the imaginary part of the electron propagator as $T \neq 0$, we obtain now the counterpart of Eq. (9) at finite temperature

$$
\begin{aligned}
d V\left(\theta, \theta^{\prime}\right)= & \tanh \left(\frac{\Lambda}{2 k_{B} T}\right) \frac{d \Lambda}{\Lambda} \\
& \times \int_{0}^{2 \pi} \frac{d \theta^{\prime \prime}}{(2 \pi)^{2}} \frac{\partial k_{\|}}{\partial \theta^{\prime \prime}} \frac{1}{v\left(\theta^{\prime}\right)} V\left(\theta, \theta^{\prime \prime}\right) V\left(\theta^{\prime \prime}, \theta^{\prime}\right) .
\end{aligned}
$$

From this point, we can reproduce all the steps following Eq. (9) for the analysis of the instabilities in the different symmetry channels. We arrive at the scaling equation including now thermal effects

$$
\begin{aligned}
\frac{\partial}{\partial \log \Lambda}\left(\begin{array}{ll}
V_{2,2} & V_{2,4} \\
V_{4,2} & V_{4,4}
\end{array}\right) \approx & \tanh \left(\frac{\Lambda}{2 k_{B} T}\right) n(\Lambda) \\
& \times\left(\begin{array}{ll}
V_{2,2} & V_{2,4} \\
V_{4,2} & V_{4,4}
\end{array}\right)\left(\begin{array}{ll}
V_{2,2} & V_{2,4} \\
V_{4,2} & V_{4,4}
\end{array}\right) .
\end{aligned}
$$

The important property of the scaling equation (25) is that, for each value of the chemical potential $\mu$, there is a value of the temperature $T_{c}$ above which the divergence in the solution disappears. We identify therefore $T_{c}$ with the critical temperature for the pairing instability. The values of $T_{c}$ obtained from the resolution of Eq. (25) have been plotted in Fig. 4. It is worthwhile to remark that, far away from the VHS, the ratio of $\Delta$ to $k_{B} T_{c}$ is very close to the BCS value of $\approx 1.76$. As the Fermi level approaches the VHS, the ratio shows however significant deviations from that value, as represented in Fig. 5. This is actually a reflection of the vanishing of the $T$-dependent gap at the critical temperature, which makes possible the probing of the proximity of the VHS at

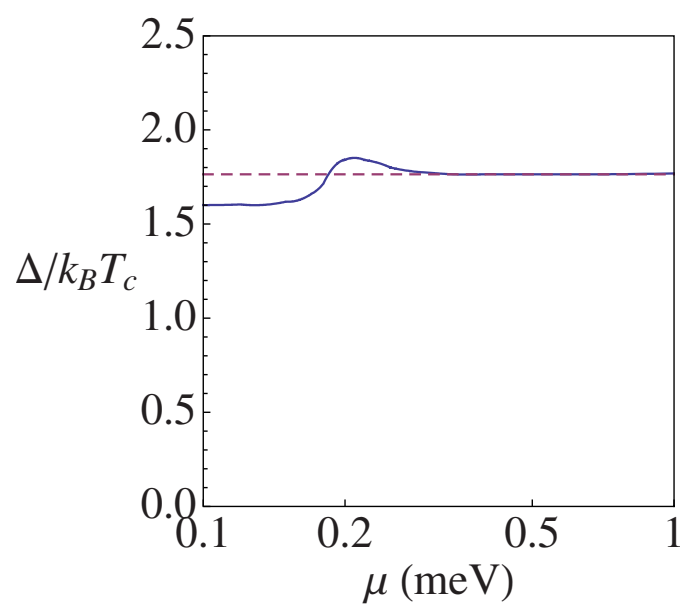

FIG. 5. (Color online) Plot of the ratio between the superconducting gap $\Delta$ and the critical temperature $T_{c}$, as a function of the deviation $\mu$ of the VHS from the Fermi level. The dashed line stands for the BCS value $\Delta / k_{B} T_{c} \approx 1.76$.

very low energies. Apart from this feature, we get the overall picture that the thermal effects play the role of reducing the strength of the pairing instability but much in the same fashion as in the more conventional electron liquids, with a value of the critical temperature in close correspondence with that of the superconducting gap.

\section{DISCUSSION}

The possibility of finding a pairing instability in graphene at temperatures of the order of $\sim 10 \mathrm{~K}$ may rely on the ability to make a fine tuning of the Fermi level to the VHS. This may be feasible as long as the proximity to the divergent density of states corresponds to a situation with a very large compressibility, which is energetically favorable. It has been shown in particular that the VHS may pin the Fermi level over a range of the chemical potential in open systems, under conditions in which the electron density is not fixed but can be balanced from the contact with a charge reservoir. ${ }^{24}$

With the experimental setups that can be devised at present, it seems possible to place the Fermi level in graphene within $1 \mathrm{meV}$ from the position of the VHS. The samples are usually doped by varying the gate voltage $V_{g}$, and typical values of the capacitance (for a 300-nm-thick $\mathrm{SiO}_{2}$ substrate) imply that a change in gate voltage of $\Delta V_{g}$ $=1 \mathrm{~V}$ should correspond to a shift in the Fermi level of $\sim 10 \mathrm{meV}$. It is therefore conceivable that by applying smaller variations in the gate voltage, and possibly using thicker $\mathrm{SiO}_{2}$ substrates with smaller capacitance, the shift in the Fermi level may be controlled with a precision below the meV scale.

The real challenge in approaching the VHS may be the characterization of samples where the divergent density of states is not smeared or attenuated due to the imperfections of the graphene lattice. In this regard, however, the theoretical estimates do not point at any scattering mechanism that can have a significant impact on the strength of the VHS. The graphene lattice may have vacancies, or even cracks and 
voids, but these give rise in general to low-energy features that are felt close to the charge neutrality point. More relevant instead may be the effect of impurities in the graphene layer. In this case, a large momentum transfer may be involved in the scattering of electrons, which is reinforced near the VHS by the approximate nesting of the Fermi line. The influence of this kind of disorder has been studied in detail in Ref. 25 in the case of the square lattice with nearest-neighbor hopping at half filling. Given that the analysis is based in essence on the nesting of the Fermi line, we can actually apply the results of that study with an appropriate translation of the relevant parameters. Thus, we arrive at the conclusion that the density of states (21) may be suppressed in general by corrections that depend on the function $\left(1 / 4 \pi t \tau_{\pi}\right) \log \left(4 \tau_{\pi}|\varepsilon|\right)$, with $t$ being the nearest-neighbor hopping and $\tau_{\pi}$ being the relaxation time related to the impurity scattering. This latter quantity can be estimated, taking into account the observation that in some graphene samples the mean-free path can be of the order of $\sim 1 \mu \mathrm{m}$. According to the value of $v_{F}$ in graphene, this may be used to estimate the relaxation time as $\tau_{\pi} \sim 10^{4} t^{-1}$. We see therefore that, at the relevant energy scales represented in Fig. 4, the corrections to the divergent density of states from impurity scattering can have a relative weight of the order of $\sim 10^{-4}$, making them insignificant in altering the strength of the superconducting instability.

Finally, we should consider also the presence of ripples as an intrinsic source of scattering in graphene. The effect of this type of disorder can be represented as a smooth modulation of the hopping parameters over the graphene lattice. We can think of this modulation as producing locally a shift in the position of the different saddle points, much in the same way as the curvature of the carbon nanotubes produces a shift of the Dirac points in the Brillouin zone. The divergence in the density of states may be then split depending on the curvature of the graphene lattice, and we have checked numerically that this is actually the case, with a scale $\Delta \varepsilon$ for the distance between the resulting VHSs which is of the order of $\sim t\left(a_{\mathrm{CC}} / R\right)^{2}$, in terms of the C-C distance $a_{\mathrm{CC}}$ and the scale $R$ of the local radius of curvature. Taking into account the change $\Delta h$ in the height of the graphene profile $(\sim 1 \mathrm{~nm})$ over a distance $L$ which is typically of the order of $\sim 10 \mathrm{~nm}$, it is easy to estimate the scale of the radius of curvature as $\sim L^{2} / 2 \Delta h \sim 50 \mathrm{~nm}$. This leads to a scale for the splitting $\Delta \varepsilon$ which is $\sim 10^{-2} \mathrm{meV}$, that is, too small to have an influence in the development of the superconducting gap when it takes place around the VHS, as observed in Fig. 4.

In conclusion, we have seen that placing graphene in the proximity of the VHS in its valence band may be a good instance to induce a superconducting instability in the electron system. The origin of this effect lies in the anisotropy of the $e-e$ scattering along the Fermi line, which leads to an attractive coupling in a channel with $d$-wave symmetry. From a practical point of view, the different sources of scattering in graphene appear to be ineffective in smearing out or attenuating the strength of the VHS. Thus, we have shown that the scale of the pairing instability may be pushed up to temperatures larger than $10 \mathrm{~K}$, depending on the ability to tune the system to the proximity of the VHS.

\section{ACKNOWLEDGMENTS}

The financial support of the Ministerio de Educación y Ciencia (Spain) through Grant No. FIS2005-05478-C02-02 is gratefully acknowledged.
${ }^{1}$ See, for instance, A. K. Geim and K. S. Novoselov, Nature Mater. 6, 183 (2007).

${ }^{2}$ K. S. Novoselov, A. K. Geim, S. V. Morozov, D. Jiang, M. I. Katsnelson, I. V. Grigorieva, S. V. Dubonos, and A. A. Firsov, Nature (London) 438, 197 (2005).

${ }^{3}$ Y. Zhang, Y.-W. Tan, H. L. Stormer, and P. Kim, Nature (London) 438, 201 (2005).

${ }^{4}$ N. M. R. Peres, F. Guinea, and A. H. Castro Neto, Phys. Rev. B 73, 125411 (2006).

${ }^{5}$ M. I. Katsnelson, Eur. Phys. J. B 51, 157 (2006).

${ }^{6}$ J. Tworzydło, B. Trauzettel, M. Titov, A. Rycerz, and C. W. J. Beenakker, Phys. Rev. Lett. 96, 246802 (2006).

${ }^{7}$ K. Nomura and A. H. MacDonald, Phys. Rev. Lett. 98, 076602 (2007).

${ }^{8}$ H. B. Heersche, P. Jarillo-Herrero, J. B. Oostinga, L. M. K. Vandersypen, and A. F. Morpurgo, Nature (London) 446, 56 (2007).

${ }^{9}$ E. C. Marino and L. H. Nunes, Nucl. Phys. B 741, 404 (2006).

${ }^{10}$ J. González, F. Guinea, and M. A. H. Vozmediano, Phys. Rev. B 63, 134421 (2001).

${ }^{11}$ B. Uchoa and A. H. Castro Neto, Phys. Rev. Lett. 98, 146801 (2007).

${ }^{12}$ A. M. Black-Schaffer and S. Doniach, Phys. Rev. B 75, 134512
(2007).

${ }^{13}$ C. Honerkamp, Phys. Rev. Lett. 100, 146404 (2008).

${ }^{14}$ R. S. Markiewicz, J. Phys. Chem. Solids 58, 1179 (1997).

${ }^{15}$ W. Kohn and J. M. Luttinger, Phys. Rev. Lett. 15, 524 (1965).

${ }^{16}$ T. Ohta, A. Bostwick, J. L. McChesney, T. Seyller, K. Horn, and

E. Rotenberg, Phys. Rev. Lett. 98, 206802 (2007).

${ }^{17}$ The factor of 3 in front of $\chi(\mathbf{0}, \omega)$ comes from the three different saddle points while the factor of 2 in front of $\chi(\mathbf{Q}, \omega)$ comes from the two inequivalent ways of connecting the saddle points.

${ }^{18}$ R. Shankar, Rev. Mod. Phys. 66, 129 (1994).

${ }^{19}$ D. Zanchi and H. J. Schulz, Phys. Rev. B 54, 9509 (1996).

${ }^{20}$ J. González, F. Guinea, and M. A. H. Vozmediano, Nucl. Phys. B 485, 694 (1997)

${ }^{21}$ J. González, F. Guinea, and M. A. H. Vozmediano, Phys. Rev. Lett. 79, 3514 (1997).

${ }^{22}$ E. Perfetto, M. Cini, S. Ugenti, P. Castrucci, M. Scarselli, M. De Crescenzi, F. Rosei, and M. A. El Khakani, Phys. Rev. B 76, 233408 (2007).

${ }^{23}$ E. M. Lifshitz and L. P. Pitaevskii, Statistical Physics (Pergamon, Oxford, 1980), Pt. 2, Chap. IV.

${ }^{24}$ J. González, Phys. Rev. B 63, 045114 (2001).

${ }^{25}$ E. P. Nakhmedov, M. Kumru, and R. Oppermann, Phys. Rev. Lett. 84, 3930 (2000). 